\title{
AOIR
}

Selected Papers of \#AolR2020:

The $21^{\text {st }}$ Annual Conference of the

Association of Internet Researchers

Virtual Event / 27-31 October 2020

\section{THE UNCERTAIN DECORUM OF ONLINE IDENTIFICATION: A STUDY IN QUALITATIVE INTERVIEWS}

\author{
Samuel DiBella \\ CyPurr Collective
}

This study explores the ethics and motivations of online identification-how and why people collect and publish identifying information about others online. In seven interviews, activists, Internet users, advocates, and journalists were asked about their investigative practice and how they viewed the ethics of deanonymization. Using ethnographic interviewing techniques and a thematic analysis inspired by grounded theory, I describe respondents 'investigations and compare them to existing theories in surveillance studies, online anonymity, and digital vigilantism. Ultimately, the interview results did not follow models of digital vigilantism and doxxing, and I caution against using those terms to apply to cases like those described in this study. I also make suggestions for how these results could augment theoretical models of anonymity, particularly how respondents ' investigative techniques and backgrounds lead them to different moral commitments.

Digital privacy debates often center around data handling by powerful actors—companies and countries. In parallel, researchers have been discussing online harassment, especially in massive, organized campaigns, and the mental-health harms they cause (Lenhart et al. 2016). But collecting identifying information is also within grasp of the everyday Internet user. A tide of digital information has made loss of anonymity an ordinary occurrence on the Internet. With social media data, public records, and tools like satellite imagery and reverse-image searching, people can easily trace the online activities of friends, acquaintances, coworkers, or strangers.

These ways of learning about people depend on unofficial sources and the unknowing complicity of others (whoever shared the information initially). In the current iteration of Internet life, there's little vocabulary for describing group privacy responsibilities or for deciding what you should do with someone else's information. The differing vulnerabilities people have online, based on gender, race, citizenship status, or economic class, make it hard to assume what will or won't harm another person (Gangadharan 2017). Asking for permission to post photos to social media is common now, even as it has become normal to look up the personal or professional social media accounts of someone you've just met. The norms for using identifying information are still evolving as Internet communities adapt digital investigative techniques to their own ends.

\section{Methodology}

To answer the questions above, I used qualitative interviews to uncover how investigators view their work, ethics, and effects. After my initial literature review, I didn't think I could Suggested Citation (APA): DiBella, S. (2020, October). The Uncertain Decorum of Online Identification: A Study in Qualitative Interviews. Paper presented at AoIR 2020: The $21^{\text {th }}$ Annual Conference of the Association of Internet Researchers. Virtual Event: AolR. Retrieved from http://spir.aoir.org. 
use theory to design the interview study-I couldn't find theories that precisely covered the area of research. Because I wanted to describe resulting data rather than infer from them, I thought an inductive analysis would work well. So, I employed the methodology of grounded theory to guide my work. With its attention to process and causality and its aim to "create" theory rather than test hypotheses (Dey 2004), I saw affinity between grounded theory and my research questions. I view my resulting study design as in line with Gabriella Coleman's $(2010 ; 489)$ call for researchers to "provincialize" culture in digital media: "Showing how, where, and why [digital media] matters is necessary to push against peculiarly narrow presumptions about the universality of digital experience."

My interviews started with a convenience sampling of respondents who were closest to my initial area of interest, doxxing and deanonymization. As interviews progressed, I used purposive sampling to fill in gaps where I thought I needed more material. For example, I made sure my later interviews included archivists and activists, because I was curious how their outlook would reflect in their practice, in comparison to the journalists I spoke with earliest. This practice is consistent with "theoretical sampling" in grounded theory, where researchers continually refine their sampling choices as they collect data (Charmaz 2006; 96).

The interviews comprised 10 hours of audio split over seven respondents. Respondents lived or worked in the United States, the United Kingdom, or Singapore, and most were journalists, hackers, activists, researchers or advocates (several in some combination). All respondents had practiced online investigations for at least several years, including investigators who identified people outside of work. Because of the duration of their practice, all investigators had adjusted their approaches to investigation and publication since they started.

\section{Results}

Investigators had a hard time balancing their moral obligation to release time-sensitive information with the lengthy work of trying to ensure no harm would result from publication. In many cases, they viewed remaining silent as unethical; they felt the knowledge they had about impending harms or the contribution they could make to government transparency compelled them to speak. But that doesn't mean they were certain about the choices they made-respondents stressed that their decisions about presentation and anonymization were case-specific. They might make different decisions, given the opportunity.

This study demonstrates the problems that arise when people collect and share personal information online. Investigators used a variety of means, including digital and physical sources, to identify people online, and they were motivated by curiosity, the requirements of their work, and their sense of justice. Their efforts to preserve anonymity were mostly confined to people peripheral to their investigation, although they also took care to prevent their work from causing online harassment. Investigators used collaboration mostly for the publication of their results, although they had difficulties with the differing technical ability of their collaborators and audiences. As a result, they were protective of how their work was interpreted and tried to avoid publishing speculation to preserve their legitimacy and prevent online harms. The small sample size limits the scope of this study, but I hope it is an indicator of fruitful directions for future research in Internet ethnography, as well as a helpful example for theories of Internet culture.

Anonymity underpins many social interactions, but it's easily revoked. The act of online identification is fraught, because of all the harms it can cause. But it's also normal on the 
Internet, since so much identifying information is freely available to those with Internet access. The infrastructure of the Internet enables lateral surveillance and sousveillance, not just mass surveillance, and the investigators I interviewed all had to adapt their existing ethical standards to this new domain. Normative ethics in privacy policies and regulation have received a lot of attention, but I hope these interviews show that applied ethics on the Internet deserves more social science study. We need to talk more about how privacy and anonymity are performed socially — what their role is and how people decide to preserve or puncture them.

\section{References}

Charmaz, Kathy. 2006. Constructing Grounded Theory. London: Sage Publications.

Coleman, Gabriella. 2010. "Ethnographic Approaches to Digital Media." Annual Review of Anthropology 39 (1): 487-505. https://doi.org/10.1146/annurev.anthro.012809.104945.

Dey, Ian. 2004. "Grounded Theory." In Qualitative Research Practice, by Clive Seale, Giampietro Gobo, Jaber Gubrium, and David Silverman, 81-94.

Gangadharan, Seeta Peña. 2017. "The Downside of Digital Inclusion: Expectations and Experiences of Privacy and Surveillance among Marginal Internet Users." New Media \& Society 19 (4): 597-615. https://doi.org/10.1177/1461444815614053.

Lenhart, Amanda, Michele Ybarra, Kathryn Zickuhr, and Myeshia PriceFeeney. 2016. "Online Harassment, Digital Abuse, and Cyberstalking in America." Data \& Society. https://datasociety.net/blog/2017/01/18/online-harassment-digital-abuse/ 